\title{
Proceso de diseño de software y proceso de diseño sonoro para un videojuego educativo
}

Software process and sound design process for an educational videogame

Recibido: marzo 10 de 2017 | Revisado: abril 14 de 2017 | Aceptado: mayo 15 de 2017

\author{
Norma LeÓn Lescano ${ }^{\mathrm{I}}$ \\ SANdra Eyzaguirre Mamani ${ }^{2}$ \\ Rosalvina Campos Pérez ${ }^{3}$
}

\section{Resumen}

El presente trabajo muestra un diseño sonoro incluido en el proceso del desarrollo de software para la creación de un set de videojuegos educativos llamado "Cultiaventura". Describe el flujo de desarrollo del mismo y hace énfasis en la importancia de incluir un modelo de diseño sonoro para asegurar la aceptación del público objetivo al cual está dirigido el videojuego. Se explica el método seguido para generar la música y efectos de sonidos, las herramientas utilizadas, los roles involucrados y los tipos de sonidos necesarios para la construcción de uno de los videojuegos educativos de la cultura Moche que incluye "Cultiaventura". El resultado obtenido es un proceso básico para incluir los efectos sonoros en un videojuego educativo dentro de un proceso de desarrollo de software.

Palabras clave: diseño, sonido, software, videojuegos, cultura Moche
1 Universidad de San Martín de Porres, Perú nleonl@usmp.pe

2 Universidad de San Martín de Porres, Perú. sandra_eyzaguirre@usmp.pe

3 Universidad de San Martín de Porres, Perú rcamposp@usmp.pe

\begin{abstract}
This research shows a sound design included in the software development process for the creation of a set of educational video games called "Cultiaventura". We describe the development flow and emphasize the importance of including a sound design model to ensure the acceptance of the target audience, which is the aimed of the videogme. We also explain the method followed to produce the music and sound effects, which tools were used, the roles involved and the kinds of sounds necessary for the construction of educational video games of the Moche culture, which includes "Cultiaventura". The result is a basic process to include sound effects in an educational video game within a software development process.
\end{abstract}

Keywords: design, sound, software, videogames, Moche culture 


\section{Introducción}

Los videojuegos educativos han demostrado ser capaces de lograr que el estudiante elabore un pensamiento crítico, resuelva problemas con creatividad y el desarrollo de habilidades para tratar problemas sociales y ambientales complejos (Sampedro, 2015). La elaboración de videojuegos educativos trae consigo la mejora de diversos métodos para la enseñanza aprendizaje de desarrollo de software interactivo, diseño gráfico, diseño sonoro, desarrollo del guion y para el propio proceso de enseñanza/aprendizaje.

El videojuego sobre el que se ha desarrollado esta investigación se llama "Correlogia", en el cual el personaje principal, el niño moche, pasa diversos obstáculos para competir con los dioses Moche. Este videojuego forma parte del grupo de videojuegos de la Cultura Moche, agrupados en "Cultiaventura". Fue desarrollado por un equipo compuesto, en su mayoría, por estudiantes de la carrera de Ingeniería de Sistemas de la Universidad de San Martín de Porres en el año 2016. Por tanto, en su construcción, se dio énfasis a la creación de las funcionalidades y librerías de código; sin embrago, se hizo sumamente importante el proceso de creación de la música y efectos de sonido.

Para la creación del sonido, se necesita de una producción independiente y paralela al desarrollo del videojuego. Su función principal consiste en soportar y realzar lo que visualmente está sucediendo (Barrios, 2007). El videojuego creado para fortalecer el conocimiento tiene un diseño sonoro adecuado a fin de mantener la atención del jugador en cada escena. Los sonidos distan de ser los sonidos de una producción cinematográfica, pues una vez finalizada la producción no vuelve a ser modificado, mientras que en el videojuego, el sonido se genera en tiempo real y en base de las acciones que el jugador va ejecutando. La probabilidad de que los jugadores puedan generar exactamente el mismo sonido es remota.

Los efectos de sonido requieren de una selección minuciosa del sonido de manera granular y un adecuado entendimiento del lenguaje que se debe expresar, debido a que son insertados en tiempos específicos en el contexto de la lógica de la acción que se está realizando. Se debe tener claro lo qué puede significar para cada persona un efecto de sonido. Por ejemplo: para un adulto, el sonido de un resorte puede significar un objeto roto, en el caso de un niño puede significar alguien saltando. Es por ello, que se debe manejar, claramente, los conceptos de significante y significado que maneja el público al que se quiere afectar.

Este trabajo muestra cómo el proceso de generación de sonidos y efectos musicales es de vital importancia en la creación de videojuegos, que amerita un proceso paralelo específico.

\section{Método}

El proceso de desarrollo del videojuego se fundamenta en la arquitectura del software y la especificación de las funcionalidades, mecánicas y directrices del videojuego que se encuentran en el documento "Game Design". Estas actividades se desarrollan en paralelo y se 
integran en la actividad de creación de código y pruebas (Figura 1). Debido a su complejidad,la actividad de creación de música y sonidos se convierte en un subproceso, el cual contiene actividades, herramientas roles y productos propios.

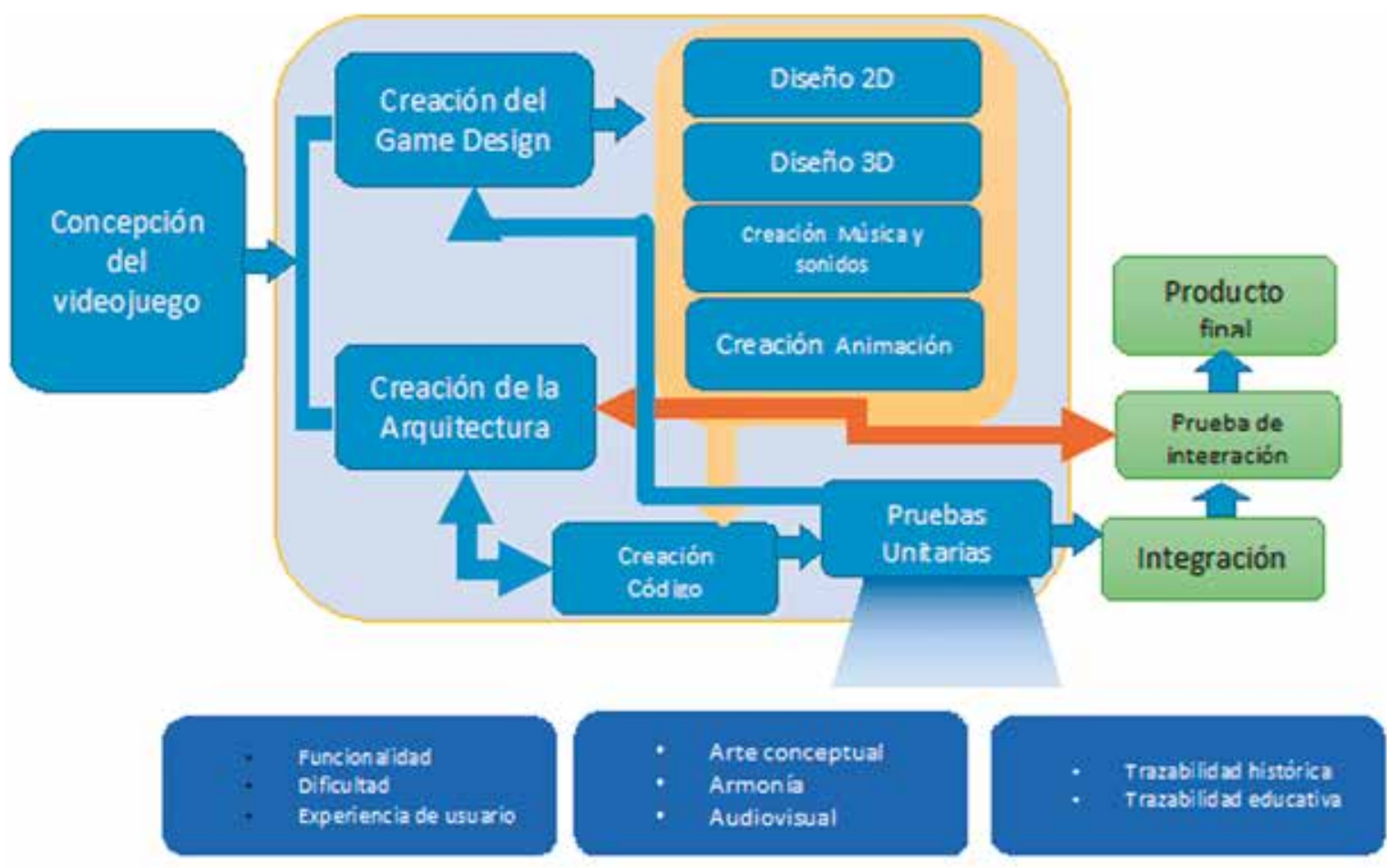

Figura 1. Proceso de desarrollo de software Fuente: (León, Eyzaguirre, \& Gomez, 2017)

El subproceso de creación de música y sonidos genera el documento de "Sonidos y música" que contiene la descripción de los diversos sonidos del videojuego y la descripción de la funcionalidad, así como el lugar y tiempo en donde incluir los sonidos. El encargado de introducir los códigos es el programador de audio (Figura 2), conocido como "Sonidista". El Game Designer y el especialista del negocio, estudian el proyecto. El Game Designer realiza la primera versión del documento "Game Design". El especialista de negocio, en este caso, un profesor y un arqueólogo desarrollan el modelo educativo para el aprendizaje mediante videojuego.

El Game Designer deriva el documento "Game Design" a los diseñadores 2d -3d, animadores y programadores. Ellos crean los diseños, animaciones, componentes, librerías, funcionalidades y código de acuerdo con el documento. Suben el archivo al repositorio GIT (servidor controlado por Visual Studio para realizar el versionamiento del código). El diseñador de audio "sonidista" obtiene el último ejecutable y designa los sonidos y música de cada parte del videojuego. Luego procede a subir los archivos al repositorio y coordina con el programador de sonidos para la colocación de los sonidos y música en el video juego. El "testeador" obtiene el último ejecutable con los sonidos y música implementados y procede a testearlo. Si encuentra un error en los sonidos y efectos musicales, se lo deriva al Game Designer y este al sonidista. 


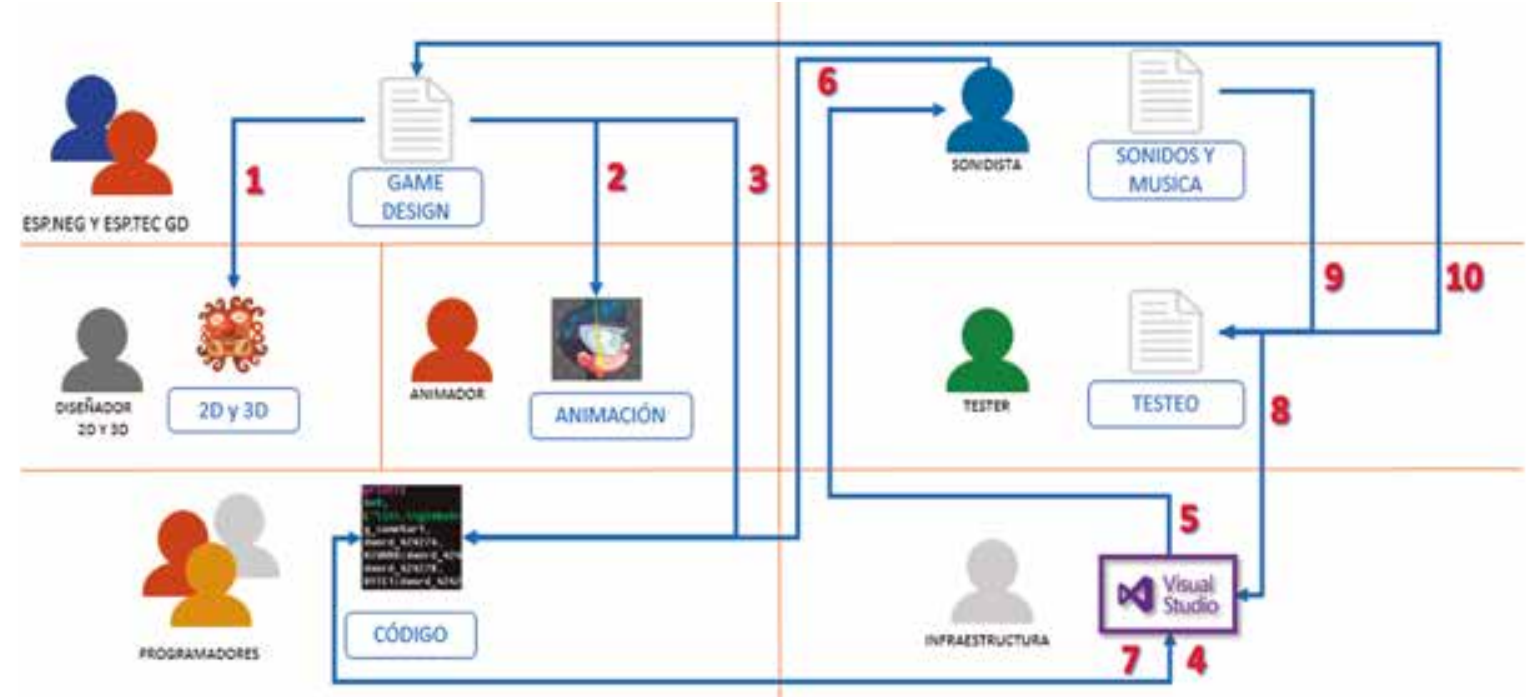

Figura 2. Documentos del proceso de desarrollo de videojuegos

\section{Herramientas para el diseño sonoro}

Las herramientas son Wise, que es un programa que facilita el diseño y la creación de audio y movimientos sofisticados durante cada fase del desarrollo del juego (AudioKinetic, 2017), y el motor mezclador FMOD Studio DSP que es un programa creación de contenido de audio para videojuegos que se caracteriza por crear sonido de alta calidad, dinámicos y flexible lo que permite una buena calidad y eficiencia (Fmod, 2017).

\section{Roles en el desarrollo de sonido}

La estructura organizativa para el desarrollo de los sonidos se acopla al pro- ceso de desarrollo de software del videojuego. Considerando que existen profesionales dedicados a distintas funciones en el proceso de creación del videojuego, se hizo necesaria la presencia de profesionales de sonidos para la creación de los sonidos y efectos. Por tanto, además, de ingenieros fue necesario trabajar con profesionales de ciencias de la comunicación y músicos para el desarrollo de los videojuegos. En este trabajo, se necesitó de un diseñador de sonido, un programador de audio (Figura 3). En proyectos más grandes, pueden existir roles adicionales como un director de audio, ingeniero de sonido e integrador de sonidos.

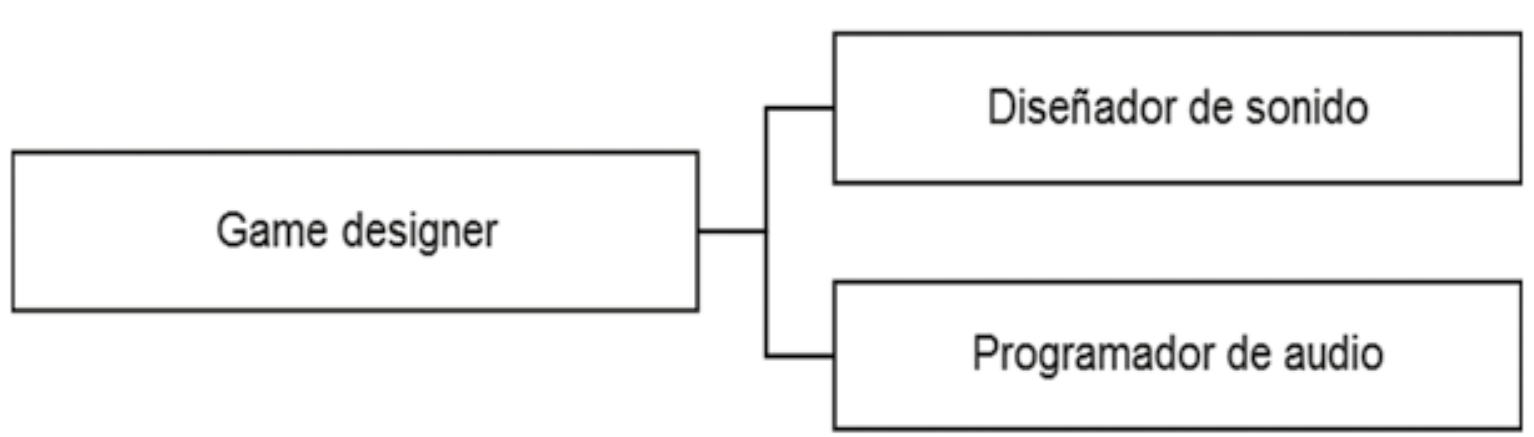

Figura 3. Roles del proceso de generación de sonido 


\section{a. Diseñador de sonido}

$\mathrm{Su}$ función es diseñar el ambiente sonoro, estilo de la música a usar, importar los sonidos directamente al motor de audio para su posterior uso, realizar las grabaciones de Foley, música, sonido ambiental y diálogos en un estudio de grabación. Elabora y ordena la lista completa de sonidos que se usarán en el proyecto, y los introduce en el banco de sonidos del proyecto, de acuerdo con el formato compatible con el motor de audio designado.

\section{b. Músico especialista en sonidos de acuerdo con el guión}

Encargado de generar la música de fondo $y$ sonidos especiales con instrumentos autóctonos de las cultura preinca de acuerdo con el guion y tema educativo (La Rosa Sanchez, 2015).

\section{c. Programador de audio}

El programador de audio es el encargado de crear las funciones, eventos $y$ procedimientos que relacionan los eventos de audio con los de video en un videojuego. En algunos casos, además, del uso de motores de audio se requiere el uso de código de programación para crear aplicaciones de audio al interior de las secuencias de programación principales que se comuniquen con el motor de audio (Rehren \& Cárdenas, 2011).

\section{Tipo de sonido para videojuegos educativos}

El diseño tiene cinco tipologías: efectos, melodías, diálogos, ambientes y efectos (Figura 4). El modelo de creación puede darse en forma paralela $\mathrm{e}$ independiente.

\section{Efectos}
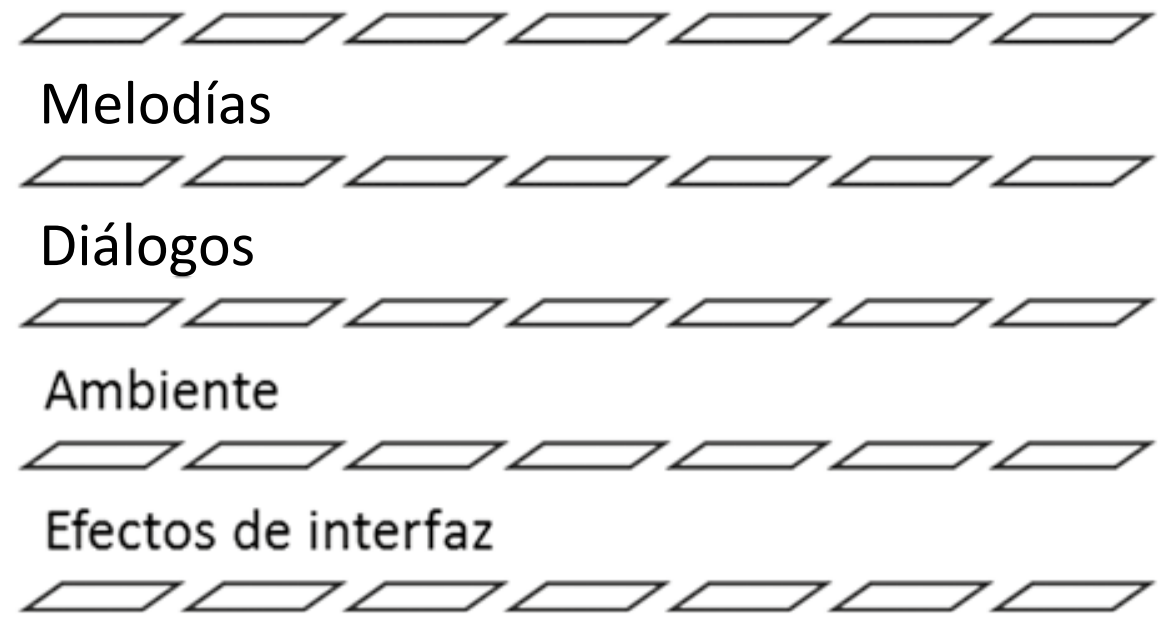

Figura 4. Tipos de sonido

\section{d. Efectos}

El efecto de sonido no lo controla el usuario. Es generado por la lógica del juego. Los efectos son desarrollados por el programador de sonidos.

\section{e. Melodías}

Son las composiciones musicales que acompañan cada interfaz del juego. Se definen de acuerdo con el público al cual va dirigido el proyecto. En este trabajo, 
las melodías fueron diseñadas para niños de Educación Básica. La melodía debe acompañar la estadía en el juego sin saturar al oyente (Figura 5). Existen ciertos pasos para la conformación de la melodía:

\section{i. Crear una sesión multipista DAW} (sistema dedicado a la grabación y edición). De esto depende el éxito de la melodía, pues al mantener los canales separados se puede influir, únicamente, en el instrumento o MIDI que se necesite corregir o cambiar.

ii. Elegir los instrumentos correctos. Esto hace referencia directamente al público, pues un instrumento sumamente grave puede aburrir al niño o consumidor de nuestro videojuego. iii. Conformar sonidos de corta duración. Hacer extensas las melodías solo conseguirá aturdir al consumidor.

iv. Manejo correcto del volumen para todo el proyecto. Si bien es cierto un proyecto correctamente mezclado no requerirá de mayores correcciones luego de ser terminado.

v. Exportar el proyecto en buena calidad. Se utiliza el formato MP3 a $320 \mathrm{Kbps}$.

vi. Elegir los espacios destinados a las melodías. Dentro del juego tenemos diversos ambientes que llevan su propia identidad y significado. Por ello, es que debemos tener claro en qué espacio específico se utilizará cada melodía y con qué fin.

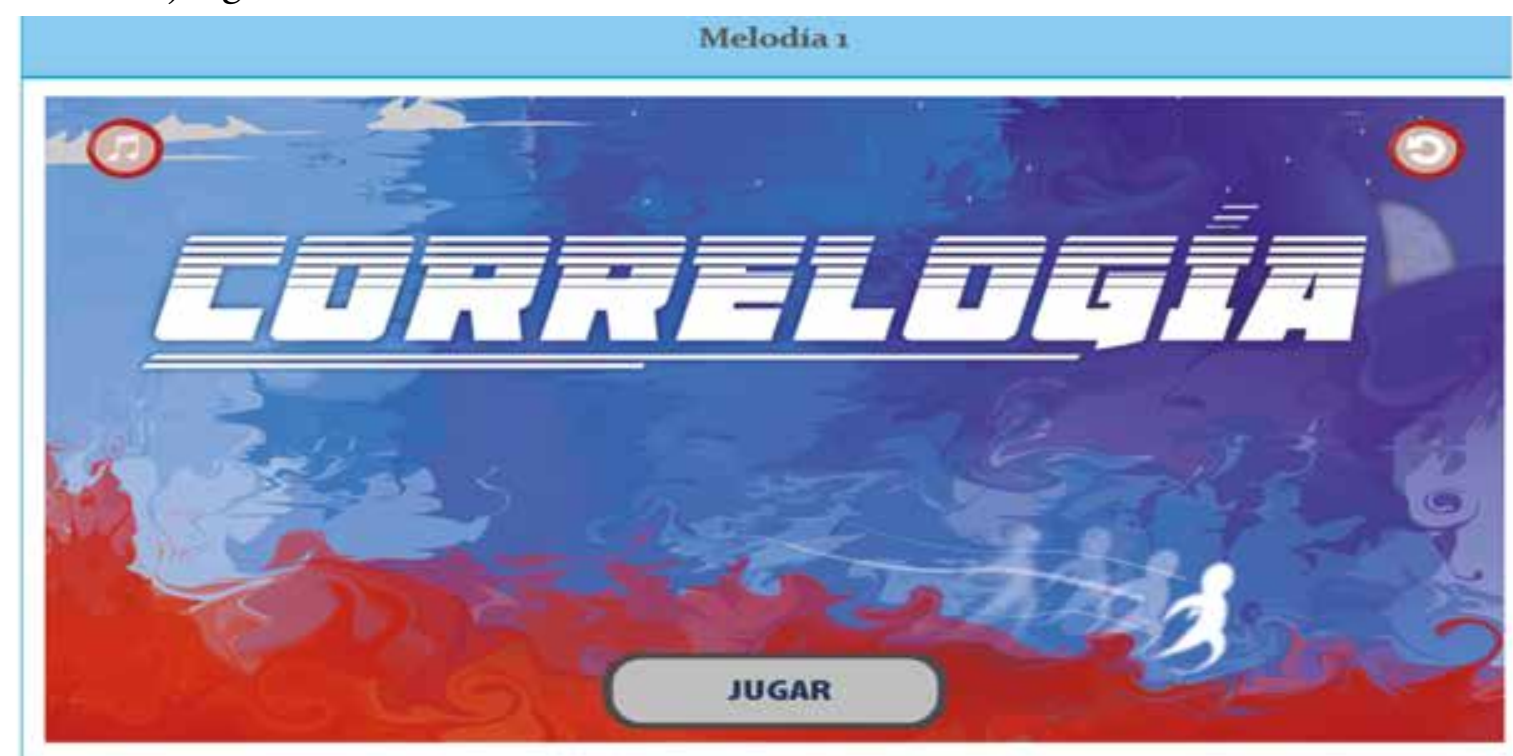

Descripción: Durante todo el mini juego se escuchará esta melodía "MCorreoi" de fondo,

Ubicación: LIA H.A.R.A/Implementación del Software/Integración y Pruebas de Software/Música y Sonidos/Cultura Moche/Archivos de música y sonidos/Organización de los sonidos y melodías según juego/4. Correlogía/melodía juego.

Figura 5. Tipos de sonido

\section{f. Diálogos}

Son conversaciones, exclamaciones, presentaciones, frases de apoyo, locuciones de explicación. Este trabajo "Cultiventura" incluye vocablos Muchik. Deben insertarse las palabras Muchik grabadas por una persona Muchik hablante. 


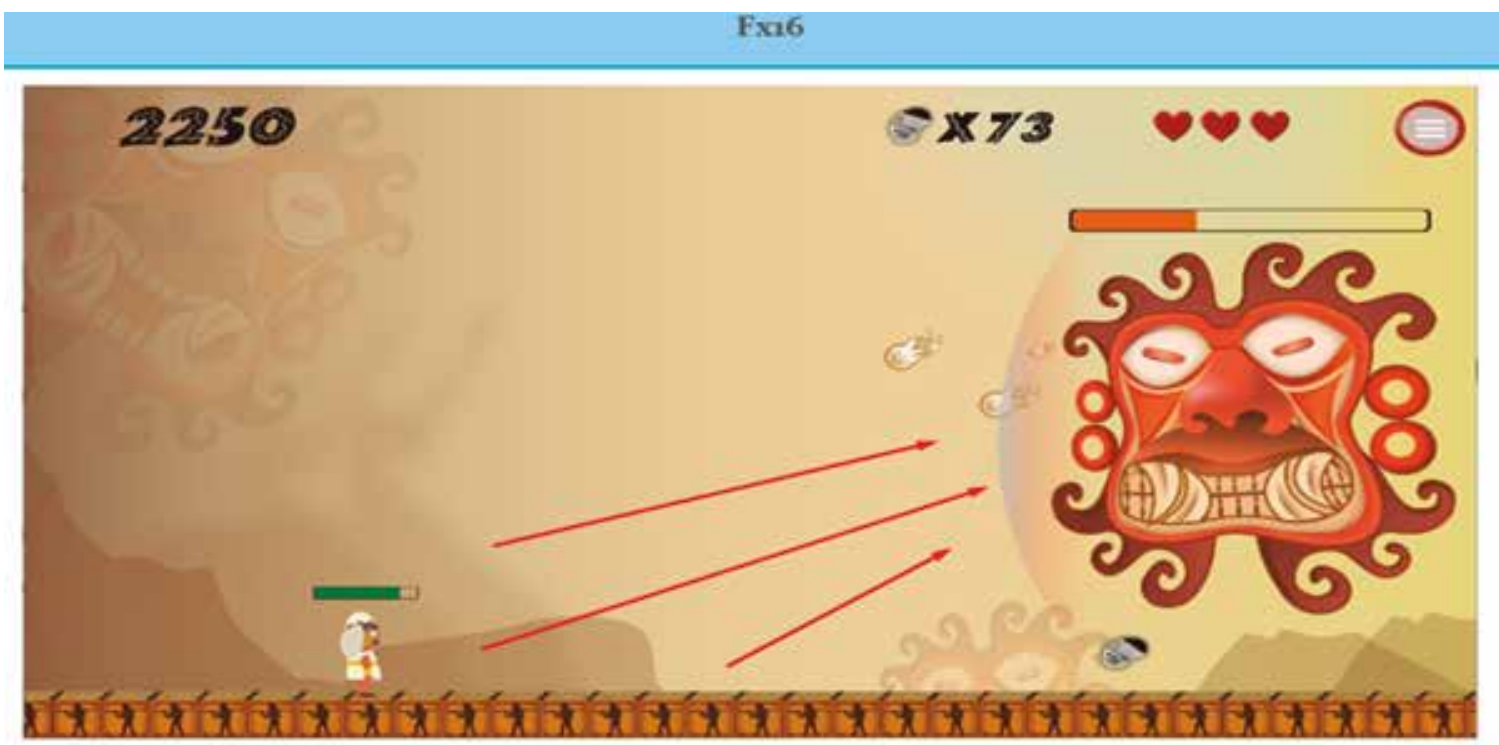

Descripción: Al botar el poder del dios, se escuchará el dialogo del Dios Aiapaec, la carga del poder,

Ubicación: LIA_H.A.R.A/Implementación del Software/Integración y Pruebas de Software/Música y Sonidos/Cultura Moche/Archivos de música y sonidos/Organización de los sonidos y melodías según juego/4. Correlogía/carga poder.

Figura 6. Tipos de sonido

\section{g. Ambientes}

Sonido característico del lugar, historia, elemento que se quiere preservar en el videojuego. Música huaco silbador.

\section{h. Efectos de Interfaz}

Efectos de objetos que el usuario manipula.

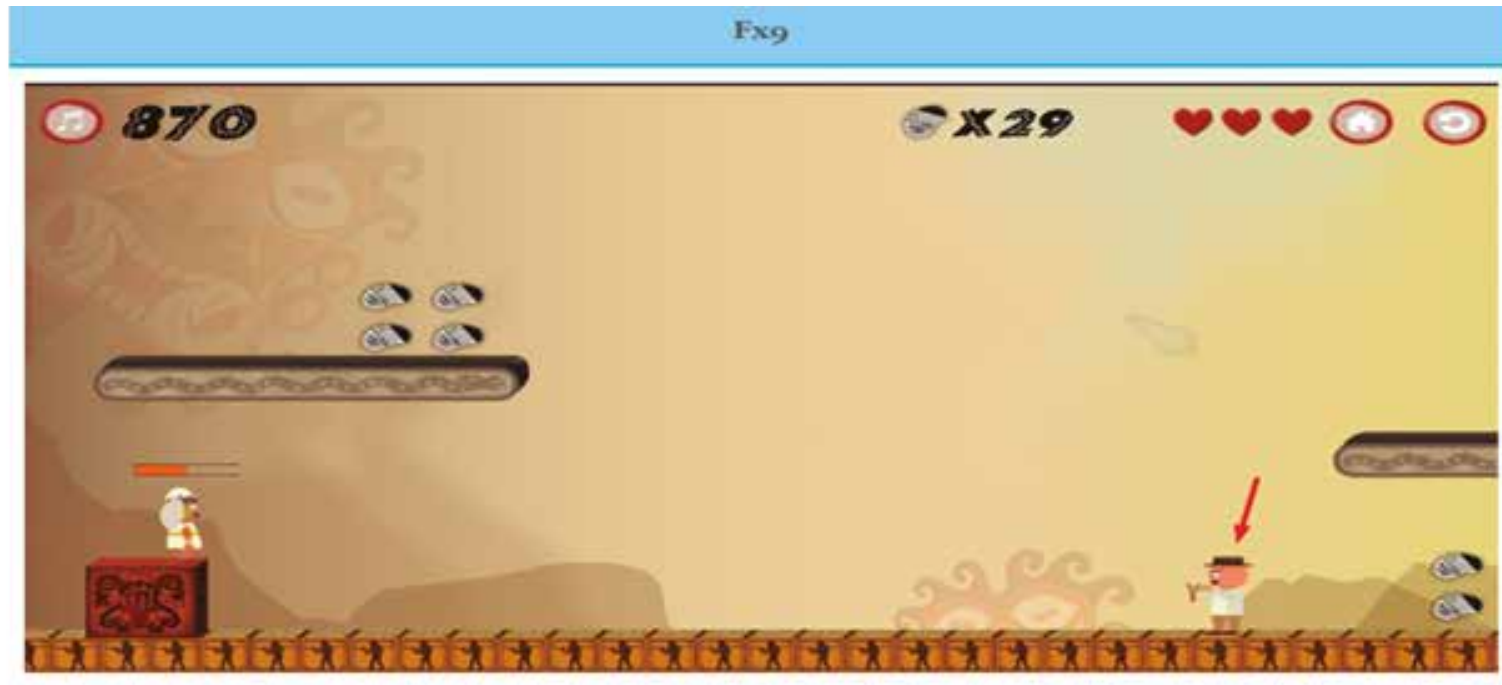

Descripción: Objeto lanzado por el huaquero

Ubicación: LIA H.A.R.A/Implementación del Software/Integración y Pruebas de Software/Música y Sonidos/Cultura Moche/Archivos de música y sonidos/Organización de los sonidos y melodías según juego/4. Correlogía/huaquero atacando.

Figura 7. Efectos de sonido 
Al jugar el videojuego de, manera simultánea, reproduce varios archivos de audio. A manera de ejemplo, y teniendo en cuenta las Figuras 6 y 7 se pueden identificar varios efectos sonoros:

1. El fuego causado por el Dios AiApaec, y el sonido de su voz

2. Deben sonar los pasos del niño Moche y debe sonar el efecto de paso de nivel.

3. El huaquero al acercarse debe hacer sentir sus pasos, su voz, así como también deben oírse sus exclamaciones o diálogos.

4. La acción ocurre en un sitio celestial; por lo tanto, debe haber ruido de ambiente celestial Moche, producido por instrumentos autóctonos Moche.

5. El escudo de protección debe generar varios sonidos como retener el ataque, el rebote del disparo. Aunque los sonidos antes, mencionados, conforman el conjunto mínimo de lo que se requiere para recrear la escena ficticia, no se puede dejar de lado la música de ambientación.

\section{Conclusión}

El proceso de elaboración de videojuegos incluye el desarrollo de música y sonidos, el cual constituye un proceso al que hay que dotar de los profesionales competentes, a fin de lograr la aceptación del aplicativo por el público objetivo.

El diseño sonoro, en la aplicación del videojuego educativo de la cultura Moche, permitió a través del sentido de la audición darle una característica propia al juego y definir ciertos parámetros de comprensión y asociación al usuario, facilitando su jugabilidad y comprensión.

\section{Referencias}

AudioKinetic. (2017). Wwise (2017.1.0). Canadá: AudioKinetic.

Barrios, A. (2007). Internet y videojuegos. Recuperado de http://datateca.unad. edu.co/contenidos/208036/Modulo_ EXE/leccin_10_diseo_sonoro_para_ video_juegos.html

Fmod. (2017). FMOD Studio. Recueprado de http://www.fmod.org/ documentation\#content/generated/ common/whatsnew_109.html

La Rosa, T. (Productor) (2015). HeartorMind Festival 2015 Opening Concert. https://www.youtube.com/ watch?v=W940JG_wWyo\&t=403s
León, N., Eyzaguirre, S. \& Gomez, J. (2017). Diseńo de entornos educativos basados en aplicaciones interactivas de realidad aumentada y videojuegos para el aprendizaje activo de la arqueología. Campus. Recuperado de http:// acceso.virtualeduca.red/documentos/ ponencias/puerto-rico/1182-838e.pdf

Rehren, C., \& Cárdenas, J. (2011). Motores de Audio para Video Juegos. Recuperado de http://mingaonline. uach.cl/scielo.php?pid=S0718025X2011000100009\&script $=$ sci_ arttext

Sampedro, B. (2015). Videojuegos para la inclusión educativa. Digital Education Review, 122-136. 